\title{
Arsenic Dimer Dynamics during MBE Growth: Theoretical Evidence for a Novel Chemisorption State of As 2 Molecules on GaAs Surfaces
}

\author{
C. G. Morgan, ${ }^{1,2}$ P. Kratzer, ${ }^{1}$ and M. Scheffler ${ }^{1}$ \\ ${ }^{1}$ Fritz-Haber-Institut der Max-Planck-Gesellschaft, Faradayweg 4-6, D-14195 Berlin, Germany \\ ${ }^{2}$ Physics Department, Wayne State University, Detroit, Michigan 48202
}

(Received 9 December 1998)

\begin{abstract}
Results of first-principles calculations are reported for the adsorption of $\mathrm{As}_{2}$ molecules on the stable surface reconstructions of the GaAs (001) surface, including adsorption paths and barriers for strongly bound sites. It is shown that a novel chemisorption state acts together with an intermediate physisorbed plateau in the total energy to hold the $\mathrm{As}_{2}$ molecules near the surface and funnel them into strongly bonding sites during epitaxial growth, and that this state can explain the transition from the $\beta 2(2 \times 4)$ to the $c(4 \times 4)$ reconstruction under low-temperature, very arsenic-rich conditions. [S0031-9007(99)09336-9]
\end{abstract}

PACS numbers: 82.65.My, 68.45.Da, 81.10.Aj

A wide variety of electronic and optoelectronic devices are based on multilayered structures composed of III-V semiconductors, such as GaAs. Understanding the fundamental kinetic processes of epitaxial growth is an important step toward optimizing the characteristics of these semiconductor layers by altering the growth conditions. Because of its technological importance as the most commonly used surface for epitaxial growth of GaAs, the GaAs (001) surface has been extensively studied. However, although the relative energies of various static reconstructions for this surface, with different surface stoichiometries, have been thoroughly studied by firstprinciples calculations $[1,2]$, gallium adatom diffusion is the only kinetic process that has been thoroughly investigated by such methods, including reaction paths and energy barriers as well as the binding energies for stable and metastable adatom sites [3]. In particular, almost nothing is known about the microscopic reaction paths, reaction barriers, and kinetics of the important processes involving incoming arsenic, such as adsorption, diffusion, and incorporation into strongly bonded sites on the surface.

Experimental work, from the earliest studies of the interaction of gallium and arsenic beams with the GaAs surface [4-6] to recent scanning tunneling microscopy studies of island shapes and size distributions immediately following a growth interruption [7], has shown that the kinetics of arsenic adsorption and attachment play an important role in $\mathrm{GaAs}$ growth. Although both $\mathrm{As}_{2}$ and $\mathrm{As}_{4}$ sources are used for epitaxial growth of GaAs, it is simpler to understand the growth processes when $\mathrm{As}_{2}$ is used, since the GaAs (001) surface reconstructions produced in standard epitaxial growth are terminated with arsenic dimers, so the $\mathrm{As}_{2}$ molecule does not necessarily have to break up in order to become incorporated into the growing surface. For both $\mathrm{As}_{2}$ and $\mathrm{As}_{4}$ sources, in order to reproduce growth without assuming unphysically large As:Ga flux ratios, the growth models used in kinetic Monte Carlo simulations commonly assume that arsenic molecules are initially adsorbed into a "molecular precursor" state [5-8].
However, no microscopic description of this precursor state has been given, and no microscopic model has been proposed for the incorporation of arsenic molecules from the precursor state into the growing surface.

GaAs growth by molecular beam epitaxy (MBE) under moderately arsenic-rich conditions produces the $\beta 2(2 \times$ 4) reconstruction, shown in Fig. 1, which must grow by filling the trenches and growing the new mountains of the next layer up. In this Letter, we report results of density-functional pseudopotential [9] calculations for $\mathrm{As}_{2}$ adsorption at a large variety of sites on the $\beta 2(2 \times 4)$ surface, with and without additional gallium atomsincluding both strongly bound sites where the arsenic dimer can attach to the dangling bonds of the surface and sites where the ad-dimer cannot bind strongly - and additional calculations on the $c(4 \times 4)$ surface, which is the stable surface produced by MBE growth under very arsenic-rich conditions.

Our calculations were carried out using both the localdensity approximation (LDA) and the generalized gradient approximation (GGA) [10]. We report the GGA results for the binding energies and adsorption barriers first, since these are known to be more reliable, followed by the LDA results in parentheses, for comparison to earlier calculations. The wave functions were expanded in a plane-wave basis with a cutoff energy of $10 \mathrm{Ry}$, and the

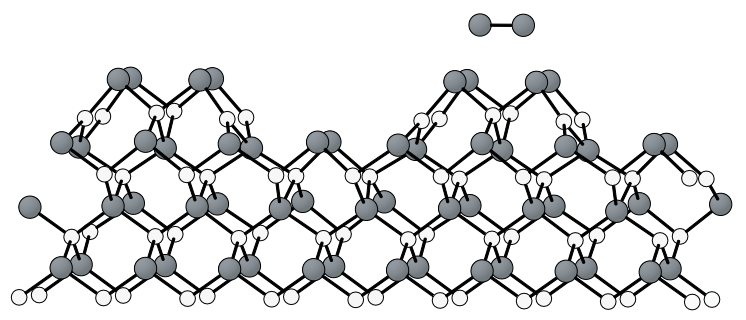

FIG. 1. The $\beta 2(2 \times 4)$ reconstruction of the GaAs $(001)$ surface with an incoming arsenic ad-dimer at the preferred "physisorbed" distance above the surface. Arsenic atoms are grey, and gallium atoms are white. 
$\mathbf{k}$-space integration was performed with a special $\mathbf{k}$-point set, with a density equivalent to $64 \mathbf{k}$ points in the Brillouin zone of the $(1 \times 1)$ surface unit cell.

We have used a slab geometry to model the ad-dimersubstrate system, with slabs containing seven or eight atomic layers, passivated on the bottom by an additional layer of pseudohydrogen atoms [11]. All the layers except the bottom atomic layer and the pseudohydrogen atoms were allowed to relax. The reported results were obtained for a $(4 \times 4)$ periodicity parallel to the surface, which we have found to be sufficient to model the interactions of an isolated $\mathrm{As}_{2}$ molecule with the surface.

When an arsenic molecule comes down on the surface, it is generally not in exactly the right position and orientation to bind immediately to the dangling bonds of the GaAs surface. Instead, we find that the molecule will initially come into a region above the surface corresponding to an intermediate plateau in the total energy, as shown in Fig. 1. In this region, the ad-dimer behaves as if it is physisorbed: it keeps the bond length of the isolated molecule ( $2.1 \AA$ ), rather than expanding to the bond length of the surface dimers $(2.5 \AA)$, and there is very little distortion of the GaAs surface below. While it remains in this region, the arsenic ad-dimer has a binding energy to the surface of about $0.2 \mathrm{eV}(0.7 \mathrm{eV}$ in the LDA). As long as the physisorbed ad-dimer is located over the mountains of the $\beta 2(2 \times 4)$ surface, it floats about $2.5 \AA$ above the surface. However, its vertical position over the trenches is not so well defined, since it can float down into the trench without encountering an energy barrier.

Estimating the lifetime $\tau$ of $\mathrm{As}_{2}$ in the physisorbed region by $1 / \tau=C \exp \left[-E_{B} /(k T)\right]$, with an attempt frequency $C=10^{13} \mathrm{sec}^{-1}$ for escape back into the vapor phase [12] and a binding energy $E_{B} \approx 0.2 \mathrm{eV}$, gives a lifetime $\tau \approx 2 \times 10^{-12} \mathrm{sec}$ during standard epitaxial growth, around $800 \mathrm{~K}$. Since physisorbed $\mathrm{As}_{2}$ molecules can move around on the surface without significantly changing their energy or causing large relaxations of the surface atoms below, they should be quite mobile, making it easier for them to find favorable binding sites on the surface and orient themselves so they can bind strongly to these sites before they desorb back into the vapor phase.

If no gallium adatoms are present on the $\beta 2(2 \times 4)$ surface, we find that the strongest binding of the arsenic ad-dimer occurs when it comes down on top of the mountain, breaking the bonds of two adjacent arsenic surface dimers and inserting itself into these bonds, as shown in
Fig. 2. The binding energy of the ad-dimer chemisorbed on the arsenic surface dimers of the mountain is about $1.6 \mathrm{eV}$ (about $2.2 \mathrm{eV}$ in the LDA); it remains practically unchanged (energy changes are less than $0.1 \mathrm{eV}$ ) when there are arsenic ad-dimers chemisorbed on the mountain in adjacent $(2 \times 4)$ unit cells, or gallium adatoms in the trench nearby.

However, a favorable binding energy alone is not sufficient to ensure that the ad-dimer actually adsorbs on this site. There must also be at least one trajectory with a small or nonexistent adsorption energy barrier for the addimer chemisorbing on this site. If the ad-dimer comes down parallel to the surface, breaking both dimer bonds simultaneously, we find that there is a prohibitively large barrier (over $1 \mathrm{eV}$, even in the LDA). However, if the ad-dimer comes down one end first, breaking one dimer bond and inserting itself, and then tips over to break the second surface dimer bond [as shown in Fig. 2(a)], there is no adsorption barrier. Therefore the site on the arsenic surface dimers of the mountain should be populated by incoming $\mathrm{As}_{2}$ molecules and depopulated by desorption of $\mathrm{As}_{2}$. The desorption is activated, with an activation energy equal to the binding energy.

Experimental work starting with the earliest studies [4-6] shows that arsenic can bind to the surface more effectively when gallium adatoms are present. Earlier theoretical work [3] has shown that the most energetically favorable place for gallium adatoms on the $\beta 2(2 \times 4)$ surface is in the trenches, and it is particularly favorable if two gallium atoms in the trench occupy adjacent sites, filling the gallium layer at the bottom of the trench and forming the $\alpha(2 \times 4)$ reconstruction in one unit cell [13].

Of all the configurations we have studied, we find the strongest binding energy, $2.4 \mathrm{eV}(3.5 \mathrm{eV}$ in the LDA), for the arsenic ad-dimer coming down on top of such a pair of gallium adatoms in the trench, and locally forming the $\beta(2 \times 4)$ reconstruction. This result is in agreement with earlier LDA calculations of Shiraishi and Ito [14], which showed that adding two gallium adatoms to fill the gallium layer at the bottom of the trench stabilizes the binding of an additional arsenic dimer in the arsenic layer above. We find that an $\mathrm{As}_{2}$ molecule coming down parallel to the surface can adsorb on top of a pair of gallium atoms in the trench with no adsorption barrier.

We find that the arsenic ad-dimer has a binding energy of $1.9 \mathrm{eV}(2.6 \mathrm{eV}$ in the LDA) on top of a single gallium atom in the trench. The arsenic atom which has no (a)

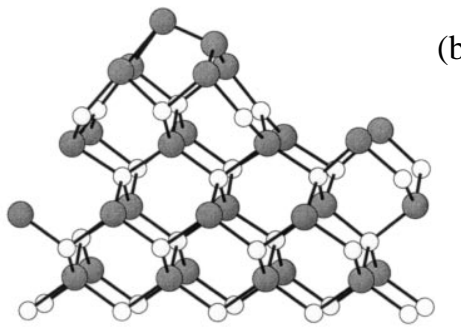

(b)

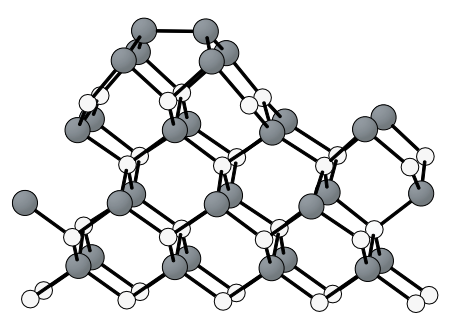

FIG. 2. (a) Arsenic ad-dimer inserting itself into the second surface dimer bond and (b) arsenic ad-dimer chemisorbed on the top arsenic layer of the $\beta 2(2 \times 4)$ surface. Arsenic atoms are grey, and gallium atoms are white. 
gallium atom beneath it will tip slightly downward toward the empty gallium site, but the dimer will not dissociate. As in all the other strongly bound adsorption sites we have investigated, the arsenic ad-dimer prefers to remain a dimer: if we separate the arsenic atoms, placing one on top of the gallium atom and the other in the empty gallium site beside, they will relax back to the tilted dimer configuration. Binding energies for the ad-dimer in other sites near gallium adatoms in the trench are less favorable than the binding energy for the ad-dimer chemisorbed on the arsenic surface dimers of the mountain.

We may estimate the desorption rate, or inverse lifetime, $D$ for arsenic ad-dimers at various strongly bonded sites on the surface by using $D=1 / \tau=C \exp \left[-E_{B} /(k T)\right]$, with an attempt frequency $C=10^{13} \mathrm{sec}^{-1}$ for escape back into the vapor phase. The binding energies $E_{B}$ and the corresponding desorption rates $D$ for an arsenic ad-dimer chemisorbed on the top arsenic layer of the mountain, or bound on top of one or two gallium adatoms in the trench, are given in Table I. We use GGA energies, rather than the overestimated LDA binding energies, in this table.

The estimated desorption rates can be compared with the incoming $\mathrm{As}_{2}$ flux during growth, e.g., $3 \times 10^{14}$ molecules $/\left(\mathrm{cm}^{2} \mathrm{sec}\right)$ [15], or 4 molecules $/ \mathrm{sec}$ falling on each $(2 \times 4)$ unit cell. Since the estimated desorption rate for an arsenic ad-dimer bound on top of a pair of gallium adatoms in the trench is very low at the commonly used temperatures for MBE growth, any ad-dimers which desorb from these sites should be easily replaced by incoming $\mathrm{As}_{2}$ molecules. However, ad-dimers bound on top of a single gallium adatom are predicted to desorb too rapidly at $800 \mathrm{~K}$ to be replaced by this incoming flux; observation of these is predicted only for higher $\mathrm{As}_{2}$ fluxes or lower temperatures. Arsenic ad-dimers chemisorbed on the top arsenic layer of the mountain are also expected to desorb too rapidly to be replaced during standard, high-temperature MBE growth around $800 \mathrm{~K}$.

Although the arsenic ad-dimers which adsorb on the mountain, on top of a single gallium adatom, or in other more weakly bound configurations near gallium adatoms are ultimately expected to desorb during growth at $800 \mathrm{~K}$, these adsorption sites still play an important role in extending the lifetime of $\mathrm{As}_{2}$ molecules on the surface. When an $\mathrm{As}_{2}$ molecule which has been temporarily adsorbed in one of these sites desorbs, it enters the physisorbed region, where it can move around reasonably freely - so it may become bound in another temporary adsorption site or find its way into a strongly bound site before it escapes back into the vapor phase. Since the $\mathrm{As}_{2}$ molecule does not dissociate in any of the adsorption sites we have found which have a binding energy of $0.5 \mathrm{eV}$ or more, these secondary adsorption sites, together with the physisorbed region, which increases the mobility of $\mathrm{As}_{2}$ on the surface, can function as a molecular precursor state, increasing the likelihood that an incident $\mathrm{As}_{2}$ molecule will become incorporated into the crystal. We note that kinetic Monte Carlo simulations of growth require a "precursor state" with a binding energy of about $1.7 \mathrm{eV}$ [16], which is quite close to the calculated binding energy for an arsenic addimer chemisorbed on the mountain. Since mountain sites should significantly outnumber unpaired gallium adatoms in the trenches, we would expect the mountain sites to play a dominant role in extending the lifetime of $\mathrm{As}_{2}$ molecules on the surface.

When the temperature is lowered, desorption rates are strongly reduced for arsenic ad-dimers at all adsorption sites. For example, if the temperature is reduced from 800 to $700 \mathrm{~K}$, the estimated desorption rate for arsenic addimers chemisorbed on the top arsenic layer of the mountain (shown in Table I) is reduced from 790 to $29 \mathrm{sec}^{-1}$. If there is no incoming gallium flux to provide sites with stronger binding energies in the trench, it seems likely that these sites can be resupplied by an $\mathrm{As}_{2}$ flux of $4.5 \times$ $10^{15}$ molecules $/\left(\mathrm{cm}^{2} \mathrm{sec}\right)$, or 60 molecules/sec falling on each $(2 \times 4)$ unit cell. So we would expect significant numbers of arsenic molecules to chemisorb and remain on the top arsenic surface layer if the surface is exposed to an $\mathrm{As}_{2}$ flux of $4.5 \times 10^{15}$ molecules $/\left(\mathrm{cm}^{2} \mathrm{sec}\right)$ at $700 \mathrm{~K}$. This suggests that under such conditions the surface should transform to a new reconstruction involving a top partial layer of arsenic chemisorbed on arsenic: the $c(4 \times 4)$ reconstruction.

The $c(4 \times 4)$ surface, which is shown in Fig. 3 with a single additional arsenic molecule chemisorbed on it, is terminated by a complete layer of arsenic, with an additional partial layer of arsenic on top. Like the arsenic atoms in the top layers on the mountain and in the trench for the $\beta 2(2 \times 4)$ surface, the arsenic atoms in the top partial layer of the $c(4 \times 4)$ surface form dimers. The local bonding for the arsenic in the top partial layer of the $c(4 \times 4)$ surface is quite similar to the local bonding for the ad-dimer chemisorbed on the top arsenic layer of the mountain, shown in Fig. 2(b): each arsenic atom in the top partial layer is bonded to its dimer partner, and

TABLE I. Arsenic ad-dimer binding energy $E_{B}$ and desorption rate $D$ (or inverse lifetime, in $\sec ^{-1}$ ) at different temperatures, for various ad-dimer sites.

\begin{tabular}{ccccc}
\hline \hline Site & $\begin{array}{c}E_{B} \\
(\mathrm{eV})\end{array}$ & $\begin{array}{c}D \\
\text { at } 500 \mathrm{~K}\left(\mathrm{sec}^{-1}\right)\end{array}$ & $\begin{array}{c}D \\
\text { at } 700 \mathrm{~K}\left(\mathrm{sec}^{-1}\right)\end{array}$ & $\begin{array}{c}D \\
\text { at } 800 \mathrm{~K}\left(\mathrm{sec}^{-1}\right)\end{array}$ \\
\hline On mountain: & 1.6 & $6.9 \times 10^{-4}$ & 29 & 790 \\
On 1 Ga in trench: & 1.9 & $6.5 \times 10^{-7}$ & 0.2 & 10 \\
On 2 Ga in trench: & 2.4 & $5.8 \times 10^{-12}$ & $4.9 \times 10^{-5}$ & $7.1 \times 10^{-3}$ \\
\hline \hline
\end{tabular}




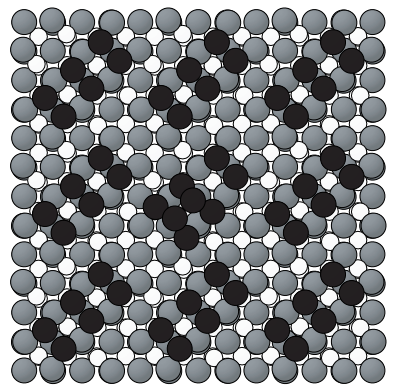

FIG. 3. One arsenic ad-dimer chemisorbed on the surface dimers of the top partial layer of arsenic for the $c(4 \times 4)$ surface. The arsenic atoms of the top partial layer and the ad-dimer are black, arsenic atoms in lower layers are grey, and gallium atoms are white. For ease of viewing, the atomic radii are taken to be larger in this figure than in the other figures.

to two adjacent arsenic atoms in the layer below. The arsenic atoms of the top partial layer and the additional chemisorbed arsenic molecule are shown black instead of grey in Fig. 3, so that it easier to see that the dimers of the top partial layer are arranged in rows of three dimers, followed by an empty dimer site.

The transition from the $\beta 2(2 \times 4)$ to the $c(4 \times 4)$ reconstruction has in fact been observed for GaAs surfaces held at $700 \mathrm{~K}$ under a somewhat lower $\mathrm{As}_{2}$ flux of $3 \times$ $10^{14}$ molecules $/\left(\mathrm{cm}^{2} \mathrm{sec}\right)$ [15]. Although we predict such a transition only at a somewhat higher flux, we cannot expect our simple method of estimating desorption rates from the calculated desorption energies to reproduce the exact boundaries for the $\beta 2(2 \times 4)$ to $c(4 \times 4)$ transition.

When an $\mathrm{As}_{2}$ molecule comes down on the $c(4 \times 4)$ surface, we find that the molecule will initially come into a region above the surface where it acts as if it is physisorbed, just as incoming $\mathrm{As}_{2}$ molecules do on the $\beta 2(2 \times 4)$ surface. While the ad-dimer remains in the physisorbed region, it has a binding energy to the surface of about $0.2 \mathrm{eV}(0.8 \mathrm{eV}$ in the LDA), and it keeps the bond length of the isolated molecule rather than expanding to the bond length of the arsenic dimers in the top partial layer $(2.5 \AA)$.

If no gallium adatoms are present on the $c(4 \times 4)$ surface, we find that the arsenic ad-dimer can bind strongly on top of two adjacent dimers in the top partial layer, by breaking these dimer bonds and inserting itself. The bonding configuration for the ad-dimer chemisorbed on these arsenic dimers looks very similar to the bonding configuration for the ad-dimer chemisorbed on the arsenic surface dimers of the $\beta 2(2 \times 4)$ surface, shown in Fig. 2(b). Since the ad-dimer on the $c(4 \times 4)$ surface is chemisorbed on top of two layers of arsenic, rather than on top of one layer, as it would be on the $\beta 2(2 \times 4)$ surface, it has a binding energy of only $1.4 \mathrm{eV}(2.2 \mathrm{eV}$ in the LDA $)$.

Figure 3 shows the top view of the $c(4 \times 4)$ surface with a single ad-dimer chemisorbed on the top partial layer of arsenic. Under sufficiently low-temperature, arsenic- rich conditions, we expect that arsenic ad-dimers will chemisorb randomly at one end or the other of each group of three surface dimers, causing the $c(4 \times 4)$ RHEED pattern to become fuzzier. If this is followed by further random arsenic adsorption at more weakly bound sites, the $c(4 \times 4)$ ordering may disappear completely. Such a change from the $c(4 \times 4)$ pattern to a disordered $(1 \times 1)$ pattern has been seen $[17,18]$ for GaAs gradually cooled from from 700 to $500 \mathrm{~K}$ under an $\mathrm{As}_{2}$ pressure of $1.5 \times$ $10^{-5}$ Torr, which corresponds to a flux of over $1.5 \times$ $10^{15}$ molecules $/\left(\mathrm{cm}^{2} \mathrm{sec}\right)$.

The enhanced chemisorption of $\mathrm{As}_{2}$ molecules on the arsenic surface dimers which occurs under lowtemperature, arsenic-rich conditions can also explain the well-documented observation that MBE growth of GaAs under such conditions leads to incorporation of up to (12)\% excess arsenic, which can produce technologically attractive, high-resistivity material with ultrashort carrier lifetimes [19].

This work was supported in part by AFOSR Grant No. F49620-96-1-0167. Cray T3E time was provided at the Fritz-Haber-Institut and the Computing Center of the Max-Planck-Gesellschaft at Garching, and by grants of time at the DOD HPC NAVO Center and at the NPACI San Diego Supercomputing Center.

[1] J.E. Northrup and S. Froyen, Phys. Rev. B 50, 2015 (1994).

[2] N. Moll et al., Phys. Rev. B 54, 8844 (1996).

[3] A. Kley, P. Ruggerone, and M. Scheffler, Phys. Rev. Lett. 79, 5278 (1997).

[4] J. R. Arthur, J. Appl. Phys. 39, 4032 (1968).

[5] J. R. Arthur, Surf. Sci. 43, 449 (1974).

[6] C. T. Foxon and B. A. Joyce, Surf. Sci. 64, 293 (1977).

[7] M. Itoh et al., Phys. Rev. Lett. 81, 633 (1998).

[8] S. V. Ghaisas and A. Madhukar, Phys. Rev. Lett. 56, 1066 (1986).

[9] M. Bockstedte et al., Comput. Phys. Commun. 107, 187 (1997); see http://www.fhi-berlin.mpg.de/th/fhimd/.

[10] J. P. Perdew, K. Burke, and M. Ernzerhof, Phys. Rev. Lett. 77, 3865 (1996).

[11] K. Shiraishi, J. Phys. Soc. Jpn. 59, 3455 (1990).

[12] An analysis of kinetic data from W. Widdra et al., Phys. Rev. B 57, 4111 (1998) shows that a prefactor as high as $10^{13}$ may be expected for the physisorption of Xe on platinum. We note that the masses of $\mathrm{Xe}$ and $\mathrm{As}_{2}$ and the $\mathrm{Xe} / \mathrm{Pt}$ and $\mathrm{As}_{2} / \mathrm{GaAs}$ interaction strengths are similar.

[13] P. Kratzer, C. G. Morgan, and M. Scheffler, Prog. Surf. Sci. 59, 135 (1998).

[14] K. Shiraishi and T. Ito, Phys. Rev. B 57, 6301 (1998).

[15] J. H. Neave et al., Surf. Sci. 133, 267 (1983).

[16] M. Itoh (private communication).

[17] K. G. Eyink et al., J. Vac. Sci. Technol. B 11, 1423 (1993).

[18] K. G. Eyink et al., J. Electron. Mater. 22, 1387 (1993).

[19] Low Temperature GaAs and Related Materials, edited by G. L. Witt et al., MRS Symposia Proceedings No. 241 (Materials Research Society, Pittsburgh, 1992). 